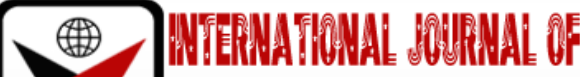

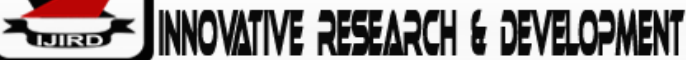

ISSN 2278 - 0211 (Online)

\section{Clothing Fit Preferences and Dressing Behaviour of Undergraduate Students: The Case of Public Universities in Ghana}

Frances Yayra Sefenu
Assistant Lecturer, Department of Fashion and Textiles Studies,
Cape Coast Technical University, Ghana
Abdul-Jaleel Saani
Lecturer, Department of Education and Psychology
University of Cape Coast, Ghana
James Amonoo
Principal Library Assistant, Sam Jonah Library
University of Cape Coast, Ghana

\begin{abstract}
:
The study investigated clothing fit preferences and dressing behaviour of undergraduate students, focusing on public universities in Ghana. The study employed descriptive cross sectional survey design. The accessible population was all undergraduate students in four premium public universities in Ghana who were 93,457 as at 2019/2020 academic year. This comprised of 57,105 (61.1\%) males and 36,352 (38.9\%) females. A sample size of 400, made up of 245 males and 155 females, was obtained. In relation to sampling procedure, the multi-stage sampling procedures were used. $A$ questionnaire, with a reliability coefficient of .827, was the instrument used. Both descriptive and inferential statistical tools were used to analyse the data. The study revealed that gender has no effect on clothing fit preferences of undergraduate students, however, in relation to dressing behaviour, it has an effect. Also, aesthetic, economic, and functional/comfort factors contributed 79.7 percent to dressing behaviour of the students. The study concludes that clothing fit preferences are able to influence positively students' dressing behaviour. The study recommends that those who matter in the University or the powers that be must establish a form of a resocialisation programme to enlighten students on the desirable dressing behaviour on campus in order for them to adopt or adapt meaningful clothing fit preferences.
\end{abstract}

Keywords: Aesthetic, clothing fit, comfort, dressing behaviour, economic, preferences

\section{Introduction}

Generally, man is considered as a social being, and has some basic needs in life such as food, clothing and shelter. Clothing is one of the most important needs of life. Clothing is seen as any skin or body covering while dressing connotes the process of act of covering the body with clothing and accessories (Alexander, Connell \& Presley, 2015). An individual's way of dressing plays an important role in enhancing his or her status in society and personality. However, if not well handled, one's dressing can damage his or her status and persona in the society. This means, socio-culturally, students dressing code is influenced by their physical body, the cultural set-up of their community, and the universe at large. Therefore, to understand the preference of students with regard to clothes that fit them, clothing retailers need some reasonable knowledge, into understanding their customers' clothing fit preference, as well as investigating into the desires of their clients in order to understand their dressing behaviour.

Sociologically, dressing that is perceived to be unfit or deviates from the societal norms, especially as it affects the religious and moral values of the society, is considered to be inappropriate. This phenomenon has influenced the clothing retail environment of university students, where demand promotes high levels of differentiation. However, the high level of dynamism in the clothing taste and preference of Ghanaian university students is creating some difficulties to clothing retailers who offer only locally Ghanaian made products (Azuah, 2017). This situation is making it difficult for retailers who offer only local products to compete effectively with their counterparts, who are offering foreign products to consumers. This competitive intensity has occasioned a need for retailers to discover the right market placement as well as generating a variation between home-made clothing products offered with that of their foreign competitors (Azuah, 2017; Essel \& Amissah, 2014).

Furthermore, by means of further global fashion products being made accessible to the Ghanaian retail markets, and with the advent of online shopping, the Ghanaian customer is developing an even greater appetite for clothing styles 
and taste that are in line with international trends (Nyang'ori, 2018). As a result, the desires of the fashion consumer are continuously explored by retailers and advertisers in order to understand their dressing behaviour, and the role consumers' gender and size play on their clothing fit preferences and dressing behaviour.

Unfortunately, it appears undergraduate students in Ghana have preference for international retail products as opposed to indigenous products (Essel \& Amissah, 2014; Laryea, 2016). As a way of helping local products to have competitive edge over international retail products, and also as a way of projecting Ghanaian identity and culture, there is the need for the youths in Ghana to develop taste and preference for indigenous retail products. This implies that local Ghanaian retailers would have to interpret global trends to help the local markets and the retailers to fashion their products to meet the socio-cultural needs of undergraduate students in Ghana, as a way of satisfying their clothing fit preferences and dressing behaviour.

The assertions so far show that undergraduate students' personal experience regarding the fitting and misfit of clothes can occasion both positive and negative dressing behaviour. Desmarteau (2017) claims that naturally they encounter discomfort when young customers feel dissatisfied with the fit of a fabric they buy in relation to their personal beliefs and negative behaviour. This dissatisfaction is not only consumer-based, but apparel manufacturers and distributors often bear the consequences when customers experience difficulties with the fitting of the clothing, such as lost sales, damage to products due to overhandling of the clothing when trying on it, as well as returned clothing due to poor fits.

Garment manufacturers and retailers in Ghana, therefore, try to fashion indigenous products to meet the standard of international retail products taking into consideration the gender and size of the clothing fits preferences and dressing behaviour of consumers. It appears these measures, notwithstanding undergraduate students in Ghana, still have preference for international retail products as opposed to locally manufactured products. Given the relevance of clothing fit preference to university students, in particular this research intends to provide research-based evidence from Ghana that looks at the issue within the context of undergraduate students in Ghana using students of public universities in Ghana in order to understand their dressing behaviour.

\subsection{Objectives of the Study}

Generally, the study investigated clothing fit preferences and dressing behaviour of undergraduate students, focusing on public universities in Ghana. Specifically, the study:

- Determined the functional/comfort, aesthetic, and economic clothing fit preferences of undergraduate students of public universities in Ghana.

- Ascertained how undergraduate students of UCC gender and size affect their clothing fit preferences and dressing behaviour.

- Examined the effects of clothing fit preferences on dressing behaviour of undergraduate students of public universities in Ghana.

\subsection{Significance of the Study}

Outcomes of the study will offer readers a better understanding of clothing fit preferences that influence the dressing behaviour of undergraduate students. The findings will also help management of the universities to encourage undergraduate students to dress in a particular way and also wear fit cloths that reflect the culture of modesty and preference in Ghana. Furthermore, the findings and recommendations that will be made in this research will help educate undergraduate students on the need to make use of information regarding clothing fit preferences and dressing behaviour provided by various sources, to reflect societal values in Ghana. Also, the findings will be useful to clothing and textiles and sewing teachers in educating students about clothing fit preferences of young consumers and their dressing behaviour.

\section{Literature Review}

Social adaptation theory of clothing formed theoretical basis for this research. This theory involves the sociocultural aspects of the thought and reasoning of a person that help in the creation of values. The theory's point is that people adapt by meaning formation and fulfilment to different positions in life (Kang-Park, 2015). The theory suggests that clothing is an expression of the inner needs of man that are conditioned by the ideals sought in clothing (Cash \& Pruzinsky, 2017). Generally, how clothing serves society is determine by the social meaning the society and individuals give to dress. Thus, consumers with positive fit preferences toward a particular clothe may end up adapting meaningful dressing behaviour towards it. Fit can be seen as how well a garment conforms to the human body in three dimensions (Desmarteau, 2017).

The fit of clothing requires visual as well as physical satisfaction of the clothes and their body function. For buyers, the most critical measurement factors to consider when buying ready-to-wear clothing are size/fit, clothing comfort and income (Sprigler, 2016).

Individuals, due to their varying body types or shapes, have different fit preferences. The perceptions of customers are influenced by several factors, including personal style preference, fashion patterns, body image and satisfaction, and attitudes (Alexander et al., 2015). Prior studies appeared to view fit as a clothing aspect that was physical and functional. The present study presumed that each undergraduate student would have a different viewpoint on appropriate clothing fit. This is so because undergraduate students within the universities found themselves in a community that is highly govern by unique social network. The students usually develop a number of acquaintances and a predominant need to belong to and to obtain recognition from a social network or peer groups (Cash \& Pruzisky, 2017). Most students try to become independent of their parents and exchange this dependence on their parents to dependency 
on the social network and peer group. Peers constitute a reference group to which the students want to belong and with whom she compares herself. In terms of dressing style and clothing, fashionable clothing is used as a group norm, indicating the dress code and how clothing are worn (Zangrillo, 2017). The peer group therefore, becomes the group from whom the student wants to receive feedback about her clothing and her general appearance in relation to norms set by current fashion.

One of the essential ways of exposing our society and its variations in other cultures is the way we dress. Clothing shows to an unseen audience the kind of person you are. Not only does dressing end with clothing, but accessories are also a form of dressing. A way of dressing may provide people with either a positive or negative view. Variables such as media, culture and community, parenting, faith, body size, and socio-economic status can influence the dressing pattern (Zangrillo, 2017).

As presented in Figure 1, the conceptual framework for the study consists of three major clothing fit preferences that collectively influence the dressing behaviour of undergraduate students. Clothing fit preferences that were considered are functional/comfort, aesthetic, and economic, and how their interaction influence the dressing behaviour of undergraduate students. Consumers' gender and body size were treated as controls since they can influence their clothing fit preferences and dressing behaviour. The argument of the study is that when clothing fit preferences are perceived positively, they can influence the dressing behaviour of consumers. However, gender and body size of consumers can influence their clothing fit preferences which in the long run may affect their dressing behaviour either negatively or positively.

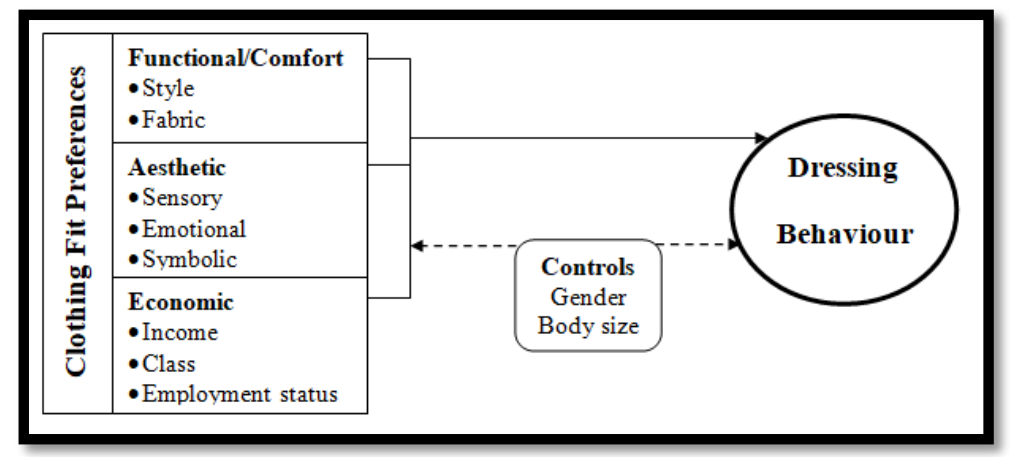

Figure 1: Influence of Clothing Fit Preferences on Dressing Behaviour of Students Source: Authors' construct (2020)

\subsection{Research Methods}

The study employed descriptive cross sectional survey design to explore the reality of clothing fit preferences of undergraduate students and their dressing behaviour, taking into consideration their gender and body size. This design created room for the researchers to examine the issues in their natural settings in order to make sense of or integrate phenomena in terms of the meanings people give to them. In relation to philosophy and approach, positivism and quantitative approach were employed respectively.

\subsection{Population, Sample and Sampling Procedure}

The target population was all undergraduate students in public universities in Ghana as at 2019/2020 academic year while the accessible population was all undergraduate students in the four premium public universities. According to Educational Management Information System (EMIS, 2020), there are 93,457 undergraduate students in the four premium public universities (Table 1). Undergraduate students from these universities were more accessible because they are the majority and have demonstrated most of the sociocultural dressing characteristics exhibited by universities students in Ghana. In relation to the subjects, they are in their transitional phase of lives; as a result, they are a target market group for many clothing manufacturers.

Using Slovin's (as stated in Saani, 2020) suggested formula, which was considered acceptable, a sample size of 400 was obtained. The formula is $n=N \div[1+N(e 2)]$, where $n$ is the sample size, $N$ is the size of the population and e is the accuracy standard. The sample size selected was appropriate since the researchers ensured that they were representative enough using the proportional sampling procedure and a recommended and tested sample formula. According to Creswell and Creswell (2018), sample size does not necessarily need to be large but how it truly represents most of the characteristics of the subjects in the population is what one must look at.

In relation to sampling procedure, the multi stage sampling procedures were used. First, purposive sampling technique was used to select four premium public universities in Ghana and level 300 undergraduate students in theses universities. The level 300 students were used for the study because they had stayed in their respective universities for some time and may have adopted fully the sociocultural dress code on campus. Also, level 300 students share similar characteristics in terms of age group, length of stay in the university, and campus experience. With regard to the subjects, stratified random sampling technique was used to select them. First, they were portioned by gender using a proportion of $.43 \%$ as presented in Table 1 . This was done in order to generate the sample for each of the strata. A proportion of .43\% was used because it represents the percentage value of the accessible population with regard to the sample size of the respondents used. After grouping the students into the various strata (university and gender) as presented in Table 1, the computer random method of simple random sampling technique was used to select the students. 


\begin{tabular}{|c|c|c|c|c|c|c|}
\hline Name of University & \multicolumn{2}{|c|}{ Male } & \multicolumn{2}{c|}{ Female } & \multicolumn{2}{c|}{ Total } \\
\cline { 2 - 7 } & PS & SS & PS & SS & PS & SS \\
\hline University of Ghana (UG) & 20,068 & 86 & 13,435 & 57 & 33,503 & 143 \\
\hline $\begin{array}{c}\text { Kwame Nkrumah University of Science and } \\
\text { Technology (KNUST) }\end{array}$ & & & & & & \\
& 13,835 & 59 & 7,450 & 32 & 21,285 & 91 \\
\hline University of Cape Coast (UCC) & 11,370 & 49 & 7,579 & 32 & 18,949 & 81 \\
\hline University for Development Studies (UDS) & 11,832 & 51 & 7,888 & 34 & 19,720 & 85 \\
\hline Total & 57,105 & 245 & 36,352 & 155 & 93,457 & 400 \\
\hline
\end{tabular}

Table 1: Accessible Population and Sample Distribution of Respondents by Gender Source: Educational Management Information System (EMIS, 2020).

Where $P S$ = population size, $S S=$ sample size

The computer random number method of simple random sampling procedure was adopted because the number of level 300 undergraduate students in each of the strata was relatively large and homogeneous. Also, this approach was used because in simple random sampling, the sampling units apart from having an equal, calculable and non-zero probability chance of being selected are independent of each other (Cohen, Manion \& Morrison, 2018). The undergraduate students were the unit of analysis for the study.

\subsection{Data Collection Instrument, Procedures and Analysis}

The method used to collect the data was a questionnaire. The questionnaire was considered acceptable because it offered a much faster way to collect data from a relatively large literate population. It also allowed anonymity of the respondents which made it easy for them to volunteer information without fear of victimisation. The Cronbach coefficient of alpha reliability obtained from the questionnaire was 0.827 . With the assistance of four field assistants, the questionnaires were administered by the researchers directly to the 400 respondents. The procedures for collecting data were carried out in two phases. The initial phase was the administering of the questionnaire while the next phases focused on retrieving the questionnaires administered. The researchers were able to retrieve all the 400 questionnaires administered in a complete form, representing $100 \%$ response rate.

Using mean and standard deviation, data on the first specific objective was analysed. Inferential statistics such as the independent samples t-test and one-way analysis of variance (ANOVA) were used to analyse data regarding the second objective. To evaluate data concerning the third objective, linear multiple regression analysis was used. These statistical tools were used because the distribution was estimated to be normal and respondents being homogeneous. The variables were also numerically calculated using discrete scales. In a variety of respects, the analysis considered ethical factors. Ethical concerns that have been raised include the right to privacy, voluntary engagement, no damage to participants, confidentiality and anonymity, deceit and scientific misconduct.

\subsection{Results and Discussion}

The rationale of the first objective was to determine functional/comfort, aesthetic, and economic clothing fit preferences of undergraduate students of public universities in Ghana. To gather data on the three facets of clothing fit preferences, several pieces of items were used. Table 2 summarizes the conclusions. The results are presented in Table 2. As per the table, respondents agreed that style (Mean $=3.874, \mathrm{SD}=.411$ ) and fabric (Mean $=3.926, \mathrm{SD}=.397$ ) are some of the functional/comfort factors (Mean $=3.900, S D=.342$ ) that ignite their clothing fit preferences. This shows that undergraduate students in the various public universities in Ghana prefer clothes that follow their body lines without any strain, identify them as distinct people, and are functional in their society. Also, the texture or quality of a fabric, the functional nature of clothes, the fibres/yarns from which the clothes are constructed, and the construction style (weaving, knitting) used in producing the clothes influence student's preference for such clothes.

\begin{tabular}{|c|c|c|}
\hline Variables & Mean & Standard Deviation (SD) \\
\hline Style & 3.874 & .411 \\
\hline Fabric & 3.926 & .397 \\
\hline Functional/comfort factors & 3.900 & .342 \\
\hline Sensory & 3.895 & .447 \\
\hline Emotional & 3.767 & .397 \\
\hline Symbolic & 3.714 & .399 \\
\hline Aesthetic factors & 3.792 & .347 \\
\hline Income & 3.661 & .387 \\
\hline Class & 3.570 & .478 \\
\hline Employment status & 3.587 & .479 \\
\hline Economic factors & 3.606 & .456 \\
\hline Clothing fit preferences & 3.766 & .391 \\
\hline
\end{tabular}

Table 2: Respondents' Views on Clothing Fit Preferences of Undergraduate Students Source: Field data (2020)

$(N=400)$ 
Also, as indicated in Table 2, respondents agreed that sensory (Mean $=3.895$, SD $=.447)$, emotional (Mean $=$ $3.767, \mathrm{SD}=.397$ ) and symbolic (Mean $=3.714, \mathrm{SD}=.399)$ are some of the aesthetic factors (Mean $=3.792, \mathrm{SD}=.347)$ that undergraduate students of public universities in Ghana consider in their clothing fit preferences. This means, colour pattern of clothes, sensitivity of the colour pattern used, the warmness of clothes, meaning assigned to particular clothes by the society, the attributes of clothes, and symbolic meanings of clothes are some of the aesthetic factors that influence students' preference for such clothes. Similarly, respondents agreed that income (Mean $=3.661, \mathrm{SD}=.387)$, class $(\mathrm{Mean}=$ $3.570, \mathrm{SD}=.478$ ) and employment status (Mean $=3.587, \mathrm{SD}=0.479)$ are some of the economic factors $(\mathrm{Mean}=3.606, \mathrm{SD}=$ .456) that persuade them to prefer some clothing fits. This shows that undergraduate students clothing fit preference is influence by their level of disposable income, cost of the cloth, price assigned to the cloth, socio-economic status, and age group.

The findings from Table 2 show that undergraduate students of the various public universities in Ghana always prefer good fit in clothing since that makes them feel confident and smart when their clothes are fit on them. They prefer casual dressing, particularly wearing jeans and a t-shirt on top because it makes them active, smart and agile. The findings are consistent with the comments of other researchers. Cloth size/fit and comfort are the two most critical measurement requirements when buying ready-to-wear clothing, according to Sprigler (2016). Also, Azuah (2017) avers that consumers perceive clothing fit positively from the comfort and aesthetic perspectives. Azuah added that there are two dimensions of clothing fit: aesthetic and functional fits that largely motivate consumers in choosing a particular cloth. Aesthetic fit refers to the appearance of the clothing in relation to the body, while functional fit refers to the comfort and functionality of the clothing because of the fit.

Furthermore, the findings are consistent with earlier researches on fit preference which found that the fit preference of customers is influenced by several variables, such as body image, body cathexis, personal comfort preference, aesthetics, current fashion trends, and lifestyle (Laryea, 2016; Sprigler, 2016).). Also, the finding on comfort is consistent with the comments of Azuah (2017) who avers that the clothing fit can be measured by both the wearer and the observer, but only the wearer can determine the comfort of the clothing. Comfort is a factor when largely perceived positive by consumers help boost their preference for such a cloth. This suggests that, when wearing well-fitted clothes, the wearer is likely to feel relaxed and confident. However, in order to better understand consumers' expectations or preferences of clothing fit and dressing behaviour, it is important to examine comfort as it relates to fit.

The second objective examined the effect of consumers' gender and body size on their clothing fit preferences and dressing behaviour. In tackling this objective, four research hypotheses were formulated and tested at .05 significant level. They are as follows:

- $\mathrm{H}_{0} 1$ : Undergraduate students' gender has no statistically significant effect on their clothing fit preferences.

- $\mathrm{H}_{0}$ 2: Undergraduate students' gender has no statistically significant effect on their dressing behaviour.

- $\mathrm{H}_{0} 3$ : Body size of undergraduate students has no statistically significant effect on their clothing fit preferences.

- $\mathrm{H}_{0} 4$ : Body size of undergraduate students has no statistically significant effect on their dressing behaviour.

In testing the first hypothesis, the independent samples t-test was conducted to compare male and female respondents clothing fit preferences and dressing behaviour. In order to examine the differences in the study constructs, the study pooled all the individual items together using the average responses to form each major variable. The results are presented in Table 3.

As depicted in Table 3, there were no statistically significant gender differences in the views of respondents with regard to functional/comfort $[\mathrm{t}=1.383, \mathrm{df}=398, \mathrm{p}=.167]$, aesthetic $[\mathrm{t}=1.457, \mathrm{df}=398, \mathrm{p}=.145]$, and economic $[\mathrm{t}=.601$, $\mathrm{df}=398, \mathrm{p}=.548]$ factors. Overall, the results show that there is no statistically significant difference between female (Mean $=3.779, \mathrm{SD}=3.246)$ and male (Mean $=3.754, \mathrm{SD}=3.288)$ undergraduate students with regard to their views on clothing fit preferences $[\mathrm{t}=1.438, \mathrm{df}=398, \mathrm{p}=.151]$. Based on this finding, the study failed to reject the first hypothesis which states that undergraduate students' gender has no statistically significant effect on their clothing fit preferences.

\begin{tabular}{|c|c|c|c|c|c|c|c|}
\hline Variable & Gender & $\mathbf{N}$ & Mean & SD & t-value & p-value & $\mathbf{\eta}^{\mathbf{2}}$ \\
\hline \multirow{2}{*}{ Functional/comfort } & Female & 155 & 3.905 & .294 & 1.383 & .167 & \\
\cline { 2 - 9 } & Male & 245 & 3.899 & .351 & & & \\
\hline \multirow{2}{*}{ Aesthetic } & Female & 155 & 3.812 & .297 & 1.457 & .145 & \\
\cline { 2 - 9 } & Male & 245 & 3.772 & .414 & & & \\
\hline \multirow{2}{*}{ Economic } & Female & 155 & 3.621 & .479 & .601 & .548 & \\
\cline { 2 - 9 } & Male & 245 & 3.591 & .524 & & & .151 \\
\hline \multirow{2}{*}{ Clothing fit preferences } & Female & 155 & 3.779 & .476 & 1.438 & & .015 \\
\cline { 2 - 9 } & Male & 245 & 3.754 & .478 & & .001 &. \\
\hline
\end{tabular}

Table 3: Gender Differences in the Views of Respondents Regarding Their Clothing Fit Preferences and Dressing Behaviour

Source: Field Data (2020) $\quad{ }^{* *} P<0.01 \quad$ Df $=398 \quad$ Where $H^{2}=$ Eta Square $\quad(N=400)$

The finding that gender has no effect on clothing fit preferences of undergraduate students, matches the observations of Laryea (2016) when he suggests that the level of importance placed on dress code and clothing in general for social activities or events varies among individuals according to their sociocultural factors and not biological factors 
such as gender. However, the finding is incongruent with the submission of Azuah (2017) who averred that the purpose of school dress codes is to minimize disruptions by eliminating the stimuli that causes distraction. When the distraction is the female body, however, girls face the undue burden of complying with gender-restrictive clothing codes that tend to sexualize their bodies by desexualizing them. Azuah found that the instructions and the justifications are unduly directed at students based on their gender, as a result the clothing fit preferences of the students are determining largely by their gender. Females usually are oppressed and marginalised through school costume code policies and their application. Similarly, the findings are inconsistent with that of Kang-Park (2015) who posits that the preference of consumers for fit is influenced by several variables, including gender.

Furthermore, the results in Table 3 show that there was a statistically significant gender difference in female $($ Mean $=3.756, \mathrm{SD}=.358)$ and male $($ Mean $=3.539, \mathrm{SD}=.388)$ respondents with regard to their dressing behaviour $[\mathrm{t}=$ $3.235, \mathrm{df}=398, \mathrm{p}=.001]$. This shows that gender has an effect on the dressing behaviour of undergraduate students. Female students indicated more positive dressing behaviour than their male counterparts. In line with this finding, the study rejects the second hypothesis which states that undergraduate students' gender has no statistically significant effect on their dressing behaviour because the evidence support otherwise. This shows that 1.5 percent of the variances in undergraduate students dressing behaviour could be explained by their gender. This implies that relatively female students' value their dressing behaviour more positively than male students. That is, female undergraduate students care more about the way they dress as compare to their male counterpart.

The finding that gender has statistically significant effect on dressing behaviour of students, and that female students have more positive dressing behaviour as compare to male students is in line per the observations of Nyang'ori (2018) who suggested that females wear clothing that arouses envy among peers and curiosity among men. In addition, the pattern of dressing and dressing behaviour of consumers can be affected by their religion and gender. Within the Ghanaian cultural context, clothing behaviours that are perceived to be indecent are usually exhibited by female students as compare to male students.

The study further examined the effect of body size on the study variables. The one-way ANOVA was conducted to explore the body size difference in the views of the respondents with regard to their clothing fit preferences and dressing behaviour. This was done to test the third and fourth hypotheses of the study at .05 significant level. The body size of respondents was treated as the independent variable while the dependent variables were respondents view on their clothing fit preferences and dressing behaviour. The ANOVA outcomes are offered in Table 4.

As depicted in Table 4, there was no statistically significant difference at .05 significant level in the views of the respondents with regard to their clothing fit preferences $[F(5,394)=3.185, p=.524]$ and dressing behaviour $[F(5,394)=$ $.209, \mathrm{p}=.812]$. This shows that the body size of consumers has no statistically significant effect on their clothing fit preferences and dressing behaviour. On the bases of the findings, the study rejects the third and fourth hypotheses. This means, undergraduate students may differ in terms of body size, however, when it comes to their dressing behaviour, they exhibit same character. This is so because the clothes and dresses are made base on the various sizes in the market, and undergraduate students dress casual to lecturers, irrespective of body size. The findings may also mean that most undergraduate students body size fall within same size such as medium to large, as a result, they all exhibit same clothing fit preferences and dressing behaviour.

\begin{tabular}{|c|c|c|c|c|c|c|}
\hline Variables & & Sum of Squares & df & Mean Square & F & Sig. \\
\hline \multirow{3}{*}{ Clothing fit preferences } & Between Groups & .688 & 5 & .229 & 3.185 & .524 \\
\cline { 2 - 7 } & Within Groups & 28.379 & 394 & .072 & & \\
\cline { 2 - 7 } & Total & 29.067 & 399 & & & \\
\hline \multirow{3}{*}{ Dressing behaviour } & Between Groups & .062 & 5 & .031 & .209 & .812 \\
\cline { 2 - 7 } & Within Groups & 58.495 & 394 & .148 & & \\
\cline { 2 - 7 } & Total & 58.557 & 399 & & & \\
\hline
\end{tabular}

Table 4: ANOVA Results on the Effect of Body Size on Respondents' Views on

Clothing Fit Preferences and Dressing Behaviour

Source: Field Data (2020)

$(N=400)$

The findings from Table 4 are inconsistent with the comments of Kang-Park (2015) who posit that consumer' worries with fit and size of clothes do differ as a result of differences in their body size. Kang-Park added that in relation to dressing behaviour, consumers who are xx-large and xxx-large prefer clothes that are loose while those who are small and medium in terms of body size prefer tight clothes. However, the results are in line with the observations of Zangrillo (2017) who submits that body size has no effect on clothing fit preferences and dressing behaviour of women. Consumers understanding of fit preferences help them to develop a proficient scheme to increase precise selection of sizes and dressing behaviour. Furthermore, the findings corroborate with that of Azuah (2017) who discovered that dressing guidelines or rules and rationales overwhelmingly target learners based on their gender and not body size. As a result, students clothing fit preferences and clothing behaviour are influenced by their gender and not body size.

The rationale for the third objective was to examine the effects of clothing fit preferences on dressing behaviour of undergraduate students of public universities in Ghana. The three facets of clothing fit preferences were treated as independent variables while the dependent variable was dressing behaviour of undergraduate students. See Figure 1 for the segregation of the study variables. To ensure that the contribution of the independent variables to the dependent variable was not due to a clear correlation between the independent variables, the collinearity diagnostic test was 
performed. The results of the analysis are presented in Table 5. As depicted in the table, all the three facets of clothing fit preferences contributed significantly to the clothing behaviour of undergraduate students. Specifically, aesthetic $(\beta=.377$, $\mathrm{p}<.01)$ contributed 37.7 percent while economic $(\beta=.242, \mathrm{p}<.01)$ and functional/ comfort $(\beta=.237$, $\mathrm{p}<.01)$ factors contributed 24.2 percent and 23.7 percent respectively.

The results from Table 5 show that aesthetic factors such as sensory, emotional, and symbolic are able to influence the dressing behaviour of undergraduate students more than the other factors. This was followed by economic factors such as income, class, and employment status. Functional/comfort factors such as style and fabric are the least factors that influence the dressing behaviour of the students.

\begin{tabular}{|c|c|c|c|c|c|c|}
\hline \multirow[t]{2}{*}{ Variables } & \multicolumn{2}{|c|}{$\begin{array}{c}\text { Unstandardised } \\
\text { Coefficient }\end{array}$} & \multirow{2}{*}{$\begin{array}{c}\begin{array}{c}\text { Standardised } \\
\text { Coefficient }\end{array} \\
\text { Beta }(\beta) \\
\end{array}$} & \multirow[t]{2}{*}{ Sig. } & \multicolumn{2}{|c|}{$\begin{array}{c}\text { Collinearity } \\
\text { Statistics }\end{array}$} \\
\hline & $\mathbf{B}$ & Std. Error & & & Tolerance & VIF \\
\hline Economic & .222 & .054 & $.242^{* *}$ & .000 & .807 & 1.239 \\
\hline Functional/comfort & .223 & .057 & $.237^{* *}$ & .000 & .753 & 1.329 \\
\hline Aesthetic & .382 & .022 & $.377^{* *}$ & .000 & .627 & 1.596 \\
\hline $\begin{array}{c}\text { Constant } \\
\mathrm{R} \\
\text { R Square } \\
\text { Adjusted R Square }\end{array}$ & \multicolumn{2}{|c|}{$\begin{array}{l}.746 \\
.893 \\
.797 \\
.784\end{array}$} & & & & \\
\hline \multicolumn{7}{|c|}{ Table 5: Effects of Clothing Fit Preferences on Dressing Behaviour of Students } \\
\hline
\end{tabular}

Overall, the results show that clothing fit preferences of undergraduate students of public universities in Ghana accounts for or influence $79.7 \%$ of the variance in their dressing behaviour. This means, quite apart from the three clothing fit preferences considered in this study, additional variables that are not taken into account in this study are likely to account for $20.3 \%$ of dressing behaviour of the students. This may mean that undergraduate students familiarise themselves to several life roles by way of value development and fulfilment such that their perception of clothing fit preferences influence their dressing behaviour. This corresponds to the argument of the social adaptation theory of clothing that posits that knowledge is absorbed into current mental structures through values and perceptions (KangPark, 2015). Thus, consumers with positive fit preferences toward a particular clothe may end up adapting meaningful dressing behaviour towards it.

The finding that undergraduate students view on functional/comfort factors contributed 23.7 percent to their dressing behaviour positively is in line with the assertion of Sprigler (2016) who posits that cloth size/fit and comfort are the two most critical measurement requirements when buying ready-to-wear clothing. Sprigler added that customers or consumers view clothing fit from two perspectives: the visual, as they look in a mirror or when they look down on themselves, and the tactile, as they feel the clothing when they wear it. This implies that every customer determines fit preference differently as to how they want a specific garment to conform to the shape of their bodies. Again, the finding that aesthetic factors such as sensory, emotional, and symbolic are able to influence 37.7 percent of students dressing behaviour corresponds to the observations of Kang-Park (2015) who avers that aesthetic factors largely influence consumers clothing fit preference and dressing behaviour. Nyang'ori (2018) also noted that comfort and aesthetic factors contribute to the dressing behaviour of consumers. Consumers in general prefer wearing clothes that will make them more confident and comfortable. Similarly, their aesthetics and economic factors influence their clothing fit preference and dressing behaviour.

\section{Conclusions}

On the whole, the research draws the conclusion that undergraduate students of public universities in Ghana perceived clothing fit preferences such as functional/comfort, aesthetic, and economic positively. That is, undergraduate students clothing fit preferences are largely influence by style, fabric, sensory, emotional, symbolic nature of the cloth, income level, class in society, and employment status. Again, the study concludes that physical fit, aesthetic fit, functional fit, social and economic comforts are able to influence the preference of students and also their dressing behaviour. However, the students differ in relation to gender with regard to their dressing behaviour but they do not differ regarding their clothing fit preferences. This suggest that undergraduate students male and female alike, are likely to contemplate not only physical fit but aesthetic and functional fit, which are affected by what they are doing and where they are going, and also their economic factors. Lastly, the study concludes that whenever economic, functional/comfort, and aesthetic factors influence undergraduate students to prefer some clothing fits, they will be able to develop positive dressing behaviour towards such products.

\section{Recommendations and Implications for Practice}

Based on the findings, the study recommends that undergraduate students should be encouraged by parents and various stakeholders to adopt or adapt dressing behaviours that reflect the culture of modesty on campus and in Ghana at large. Also, lecturers in the area of Clothing and Textiles/Sewing with the help of management of the universities can begin to factor in dressing culture orientation and socialisation in the universities' orientation programmes. This will help educate fresh undergraduate students on clothing fit preferences and attributes they should look out for during their 
clothing selection, good dress sense and modesty. This education when effectively provided will make them more concerned and objective about the clothing fit preferences they should look out for in their selection of clothing and dressing behaviours in general. The education can be extended to clubs and associations within the university.

Also, management of the universities should introduce a bi-semester based orientation activities to educate students about the right option of campus apparel and dressing behaviour on campus as a whole in order for them to adopt or adapt meaningful clothing fit preferences. Also, seminars on different clothing styles and the necessary opportunities or occasions to match should be held. Seminars on the ideals of individuals and their effect on society should be carried out. In order to exchange similar viewpoints, including those on their clothing fit preferences and dressing behaviours, students should be allowed to join programmes from other departments. In addition, differences among students with regard to gender should be considered when drafting dressing code policy of the universities. Parents may also help in socialising their children at that level whenever they come home for holidays or break with regard to the symbolic nature of clothes, aesthetic fit, comfort fit and their economic fit. This form of socialisation may help in transferring Ghanaian clothing values to the youth to maintain appropriate dressing behaviours on campus and in the country as a whole.

Again, students' associations and organisations in the various public universities should be encouraged to elect culture ambassadors as part of their executives to help educate and monitor the choice of clothing and the fit preferences of their colleagues to reflect the dressing values of decency upheld in Ghana. Also, being global minded is very characteristic of young academics, yet, the accepted way of dressing in Ghana should not be sacrificed at the expense of unaccepted clothing practices in other jurisdictions. Hence, the need for a collaborative effort by institutional management and international relation experts to support the education of decent fit preferences and dressing on various university campuses.

\section{Delimitation and Limitations}

The study was delimited to undergraduate students of public universities in Ghana. In relation to variables, the study was delimited to variables such as clothing fit preferences, gender, body size, and dressing behaviours of undergraduate students. The study purposively considered only level 300 students of four premium universities in Ghana. Therefore, responses given by this particular group of students may not represent the views of undergraduate students in Ghana, as a result, care must be taken when generalisation the findings. Also, some students may choose not to participate in this study because of personal reasons or because of the study's optional participation clause. The clothing behaviour measured here has not been validated against actual observations of the subjects' clothing behaviour and may vary from it to some extent. There may be limitations to the validity of other measures used. The conformity scale, since it was separated from the entire clothing fit preference inventory, may be questioned on that basis. Attitude measures are at best tenuous and somewhat subjective, particularly when used in a new field.

\section{References}

i. Alexander, M., Connell, L. J., \& Presley, A. B. (2015). Clothing fit preferences of young female adult consumers. International Journal of Clothing Science and Technology Journal, 37(1), 52-63.

ii. Azuah, S. (2017). Factors influencing Takoradi Polytechnic students' choice of clothing. Unpublished master's thesis, Department of Vocational and Technical Education, College of Education Studies, University of Cape Coast, Cape Coast.

iii. Cash, T. F., \& Pruzinsky, T. (2017). Body image: A handbook of theory, research, and clinical practice (3 ${ }^{\text {rd }}$ ed.). New York, NY: The Guilford Press.

iv. Cohen, L., Manion, L., \& Morrison, K. (2018). Research methods in education (8th ed.). New York: Routledge.

v. Creswell, J. W., \& Creswell, J. D. (2018). Research design: Qualitative, quantitative and mixed methods approach. London, UK: SAGE Publications.

vi. Desmarteau, K. (2017). Let the fit revolution begin. Bobbin, 72(2), 42-56.

vii. Educational Management Information System [EMIS] (2020). Public universities' enrolment by gender and programme: Final draft. Unpublished report, GES/MoE, Accra, Ghana.

viii. Essel, O. Q., \& Amissah, E. K., (2014). In search of Ghana's appropriate modern dress code. Methodology, 20(1), 12-29.

ix. Kang-Park, J. (2015). Consumers' satisfaction with ready-to-wear apparel products: Comparison of misses-sized and petite-sized women. Unpublished doctoral dissertation. University of Minnesota, St. Paul, Minnesota.

x. Laryea, D. A. (2016). Social cognition and the study of clothing and human behaviour in Ghana. Accra, Ghana: Black-Blue Press.

xi. Nyang'ori, E. A. (2018). Factors influencing consumers' selection of imported over local clothing among women in Nairobi, Kenya. Unpublished master's thesis, Kenyatta University, Nairobi.

xii. Saani, A-J. (2020). Introduction to research methods in education. Unpublished lecture synopsis, Department of Education and Psychology, Islamic University College, Ghana, Accra.

xiii. Sprigler, M. J. (2016). The aesthetics of personal style: The interaction between fashion and interiors. Unpublished master's dissertation, University of Kentucky, Kentucky.

xiv. Zangrillo, F. L. (2017). Fashion design for the plus-size (2nd ed.). New York, NY: Fairchild Publications. 